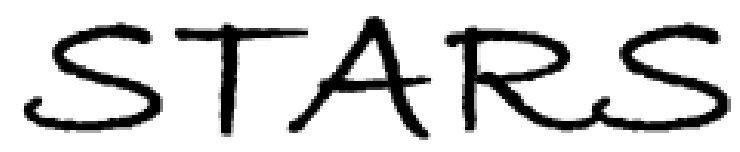

University of Central Florida

STARS

$1-1-2008$

\title{
Effect of laminar velocity profile variation on mixing in microfluidic devices: The sigma micromixer
}

\author{
Ehsan Yakhshi Tafti \\ University of Central Florida \\ Ranganathan Kumar \\ University of Central Florida \\ Hyoung J. Cho \\ University of Central Florida
}

Find similar works at: https://stars.library.ucf.edu/facultybib2000

University of Central Florida Libraries http://library.ucf.edu

This Article is brought to you for free and open access by the Faculty Bibliography at STARS. It has been accepted for inclusion in Faculty Bibliography 2000 s by an authorized administrator of STARS. For more information, please contact STARS@ucf.edu.

\section{Recommended Citation}

Tafti, Ehsan Yakhshi; Kumar, Ranganathan; and Cho, Hyoung J., "Effect of laminar velocity profile variation on mixing in microfluidic devices: The sigma micromixer" (2008). Faculty Bibliography 2000s. 1044.

https://stars.library.ucf.edu/facultybib2000/1044

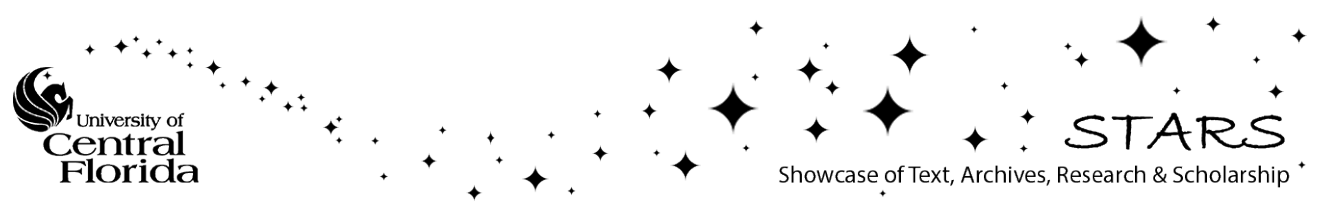




\section{Effect of laminar velocity profile variation on mixing in microfluidic devices: The sigma micromixer}

Cite as: Appl. Phys. Lett. 93, 143504 (2008); https://doi.org/10.1063/1.2996564

Submitted: 15 April 2008 . Accepted: 08 September 2008. Published Online: 10 October 2008

Ehsan Yakhshi Tafti, Ranganathan Kumar, and Hyoung J. Cho

\section{ARTICLES YOU MAY BE INTERESTED IN}

Rapid laminating mixer using a contraction-expansion array microchannel

Applied Physics Letters 95, 051902 (2009); https://doi.org/10.1063/1.3194137

A "twisted" microfluidic mixer suitable for a wide range of flow rate applications

Biomicrofluidics 10, 034120 (2016); https://doi.org/10.1063/1.4954812

Experimental test of scaling of mixing by chaotic advection in droplets moving through microfluidic channels

Applied Physics Letters 83, 4664 (2003); https://doi.org/10.1063/1.1630378

\section{Applied Physics Letters}

Mid-IR and THz frequency combs special collection 


\title{
Effect of laminar velocity profile variation on mixing in microfluidic devices: The sigma micromixer
}

\author{
Ehsan Yakhshi Tafti, Ranganathan Kumar, and Hyoung J. Cho ${ }^{\text {a) }}$ \\ Mechanical Material and Aerospace Engineering, University of Central Florida, 4000 Central Florida \\ Blvd., Orlando, Florida 32816, USA
}

(Received 15 April 2008; accepted 8 September 2008; published online 10 October 2008)

\begin{abstract}
The effect of the laminar velocity profile and its variation on mixing phenomena at the reduced scale is studied. It is shown that the diffusive mass flux between two miscible streams, flowing laminar in a microchannel, is enhanced if the velocity at their diffusion interface is increased. Based on this idea, an in-plane passive micromixing concept is proposed and implemented in a working device (sigma micromixer). This mixer shows considerable mixing performance by periodically varying the flow velocity profile, such that the maximum of the profile coincides with the transversely progressing diffusion fronts repeatedly throughout the mixing channel. (c) 2008 American Institute of Physics. [DOI: 10.1063/1.2996564]
\end{abstract}

Mixing is one of the key unit operations for laboratoryon-a-chip bioanalysis systems where often quick and efficient mixing of reactants is required. ${ }^{1,2}$ Nevertheless, mixing is greatly limited at the microscale ${ }^{3,4}$ where fluid flow is usually laminar. Transport processes at reduced scales are mainly due to the random motion of the molecules, i.e., diffusion-based processes, and not due to inertial effects. Accordingly, the dominating process in mixing is also molecular diffusion.

The effect of velocity on diffusion across an interface is explained as follows. Consider the diffusion of a species (A), diffusing from a constant source into a liquid film (B), flowing with a given velocity profile (Fig. 1). The simplified transport equation for the concentration of $\mathrm{A}$ in film $\mathrm{B}, c_{\mathrm{A}}$, can be written as follows with $D_{\mathrm{A}, \mathrm{B}}$ being the binary diffusion constant: ${ }^{5}$

$$
v_{\max } \frac{\partial c_{\mathrm{A}}}{\partial z}=D_{\mathrm{A}, \mathrm{B}} \frac{\partial^{2} c_{\mathrm{A}}}{\partial x^{2}}
$$

with the boundary conditions as $c_{\mathrm{A}}=0$ (at $\left.z=0\right), c_{\mathrm{A}}=c_{0 \mathrm{~A}}$ (at $x=0)$, and $\partial c_{\mathrm{A}} / \partial x=0(x=\delta)$. Using a similarity variable approach, the solution has the following form:

$$
\begin{aligned}
\frac{c_{\mathrm{A}}}{c_{0 \mathrm{~A}}} & =1-\frac{2}{\sqrt{\pi}} \int_{0}^{x / \sqrt{4 D_{\mathrm{A}, \mathrm{B}} z / v_{\max }}} \exp \left(-\xi^{2}\right) d \xi \\
& =\operatorname{erfc}\left(\frac{x}{\sqrt{4 D_{\mathrm{A}, \mathrm{B}} z / v_{\max }}}\right) .
\end{aligned}
$$

The local mass flux at the interface may be found as follows:

$$
N_{\mathrm{A}, x, \text { interface }}(z)=-D_{\mathrm{A}, \mathrm{B}} \frac{\partial c_{\mathrm{A}}}{\partial x_{x=0}}=c_{0 \mathrm{~A}} \sqrt{\frac{D_{\mathrm{A}, \mathrm{B}} v_{\max }}{\pi z}} .
$$

It is observed from the expression given above that increasing the magnitude of the velocity at the interface $\left(v_{\max }\right)$ translates into enhancement of the diffusive mass flux across the interface. This is a key idea for the proposed mixing concept.

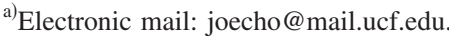

Going back to the original mixing problem in a micromixer where two miscible liquids flow side by side in a laminar regime, two diffusion fronts would be developed in a steady situation. ${ }^{6}$ These diffusion fronts progress in the transverse direction of the flow as one moves downstream in the channel. Based on Einstein's relationship for Brownian motion, ${ }^{7}$ the position of the interfaces progresses in the transverse direction to the flow as follows:

$$
x^{\prime}(z)=\left(2 D_{\mathrm{A}, \mathrm{B}} \tau\right)^{1 / 2}=\left(2 D_{\mathrm{A}, \mathrm{B}} z / U\right)^{1 / 2},
$$

where $x^{\prime}, \tau$, and $U$ are displacement of the interface from the centerline, channel residence time, and average fluid velocity, respectively.

Two major facts of this work are established as follows.

(1) Mass flux at the diffusion interface between two species enhances as the interface velocity is increased.

(2) Diffusion interfaces progress in the transverse direction of the flow as one moves downstream through the channel.

Based on these two facts, a passive mixing concept is pro-

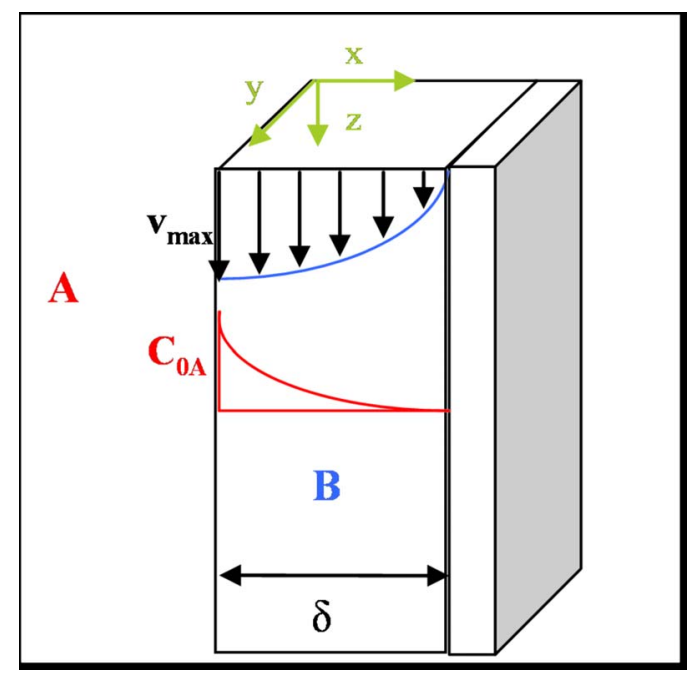

FIG. 1. (Color online) Species A diffuses into liquid film B flowing with a given velocity profile. The interface concentration limit is $c_{0 \mathrm{~A}}$ (Ref. 5). 


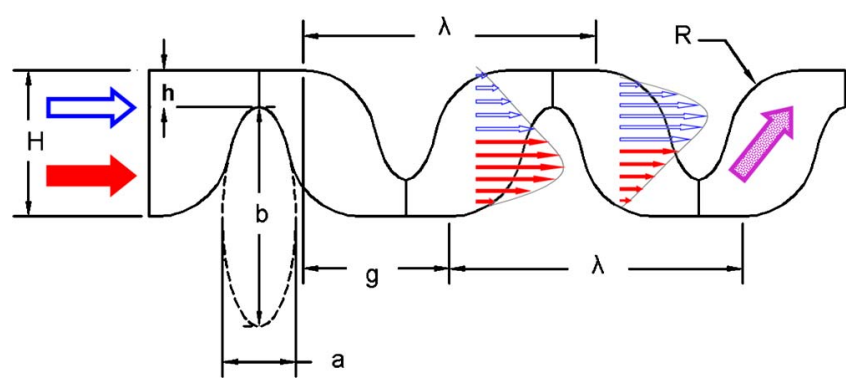

FIG. 2. (Color online) Velocity profile varies throughout the channel periodically due to the sidewalls. The maximum of the profile shifts from the center and periodically sweeps the transverse direction, overlapping the progressing diffusion fronts which results in the enhancement of diffusion.

posed that performs based on flow profile variation. This variation is due to the sidewall design that causes the maximum of the velocity profile $v_{\max }$ to be shifted or rather "swept" periodically across the transverse direction throughout the mixing channel such that it overlaps the transversely progressing diffusion interfaces (fronts) repeatedly (Figs. 2 and 3). With respect to fact (1), the overall mass flux at the interfaces would increase due to higher velocities. This is in contrast with the conventional $\mathrm{T}$ mixer that has a constant parabolic velocity profile, where the maximum occurs only at the centerline. As the diffusion fronts move out transversely away from the centerline, they coincide with the slower moving lamellae of fluid. Lower diffusion interface velocities result in rapid reduction of the diffusive flux and hence poor mixing in the conventional $\mathrm{T}$ mixer.

The sidewall profiles of the sigma mixer are generated by a combination of periodic circular and ellipsoidal arc features; "spline" and "cosine" features can also be used. Note that the upper and lower wall profiles of this design, unlike other serpentine or wavy channels, repeat periodically with a phase lag causing nonsymmetric channel width variation. As shown in Fig. 2, parameters $a$ and $b$ define the ellipsoid used to generate the side wall patterns. They are chosen such that asymmetric sidewalls are generated with least parallelism and that the maximum variation of the velocity profile is achieved passively. $\lambda$ is the periodic length of the repeating sidewall profiles and $g$ is the lag between them. The parameter $g$ being too short or too long, keeping other parameters constant, results in sidewall interference or parallel walls. $H$ is predefined as channel width and $h$ is the constriction width that together with other parameters makes the sidewalls expand or contract asymmetrically; if chosen too small, then large pressure drops would occur. Sharp corners are removed by smoothening the walls to ensure that no dead volumes are created in the device $(R)$. Numerical simulations resulted in optimized values of $H=200, h=50, a=300, b=100, R=100$, $\lambda=400$, and $g=200$ with channel depth of 100 (unit: micrometer).

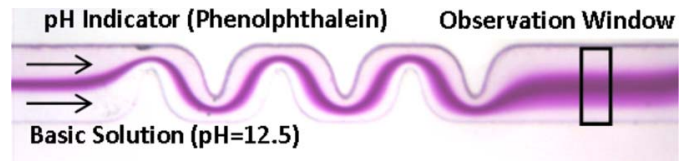

FIG. 3. (Color online) Evaluation of mixing performance using color changes as a result of mixing of a $p \mathrm{H}$ indicator (phenolphthalein) and a basic solution (sodium hydroxide, $p \mathrm{H}=12.5$ ).
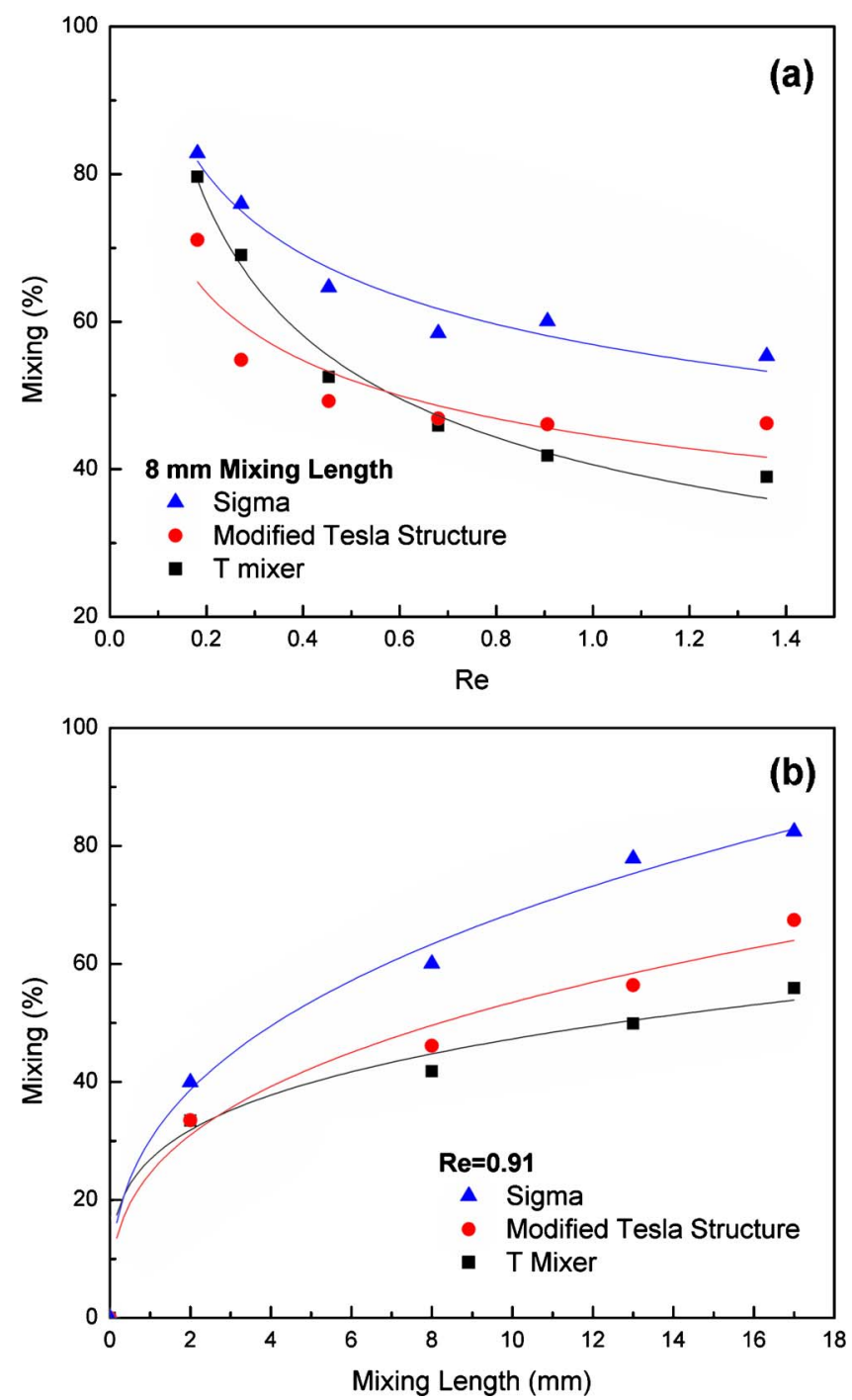

FIG. 4. (Color online) (a) Mixing performance at a given mixing length $(L=8 \mathrm{~mm})$ as a function of flow Reynolds number, (b) Mixing performance at a given flow rate $(Q=1000 \mu \mathrm{l} / \mathrm{h}, \operatorname{Re}=0.91)$ as a function of mixing length.

In order to evaluate the mixing performance, a reported method based on color changes resulting from $p \mathrm{H}$ indicators was adopted. ${ }^{1}$ Phenolphthalein solution (phenolphthalein $1 \%$, isopropyl alcohol $60 \%$, water balance) and $0.4 \mathrm{~N}$ sodium hydroxide solution $(1.6 \%$ sodium hydroxide in a similar solution to above and $p \mathrm{H}=12.5)$ were used. The two colorless solutions are introduced at the inlets of the devices by

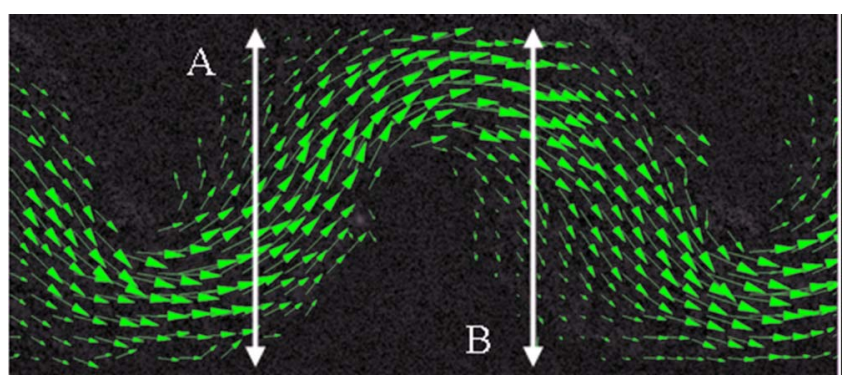

FIG. 5. (Color online) Microparticle image velocimetry measurement of the laminar flow. Bottom layers move faster than the top layers at section A, whereas in section B the situation is reversed confirming passive variation of the velocity profile. 
an infusion pump. When mixed, a pink/purple region is created which expands transversely with two interfaces away from the centerline as the solutions further mix downstream (Fig. 3). Color images ${ }^{8}$ at select cross sections are converted into thresholded grayscale images. Dark pixels, gray intensity $I=0$, show fully mixed regions whereas bright pixels, $I$ $=1$, are nonmixed portions of the flow. Standard deviation of gray intensity around the darkest gray pixel (completely mixed region in the middle of the channel) is calculated for the pixels of the selected cross section. ${ }^{9}$

$$
\sigma=\left[\frac{1}{n} \sum_{i=1}^{n}\left(I_{\min }-I_{i}\right)^{2}\right]^{1 / 2} .
$$

$n$ is the number of the cross section pixels, $I_{i}$ is gray intensity of pixel $i$ of the selected cross section, and $I_{\min }$ is the minimum gray intensity. When there is no mixing, the standard deviation, $\sigma=1$, and ideally at full mixing it will reach 0 as all pixels attain uniform gray intensity. The level of mixing (percentage) is defined as follows:

$$
M=(1-\sigma) \times 100 .
$$

The mixing performance was evaluated in comparison to the $\mathrm{T}$ mixer and the Tesla mixer ${ }^{1,2}$ as a function of flow Reynolds number and mixing length [Figs. 4(a) and 4(b)].
The result validates the effectiveness of the proposed passive micromixing concept and improvement of mixing performance over the existing designs. Finally, microparticle image velocimetry was utilized for visualization of the flow. Figure 5 confirms that the velocity profile variations occur as predicted.

This work has been supported by the National Science Foundation (ECS-0348603). The authors acknowledge technical support from Korea Bio-IT Foundry Center at the Seoul National University, Korea.

${ }^{1}$ R. H. Liu, M. A. Stremler, K. V. Sharp, M. G. Olsen, J. G. Santiago, R. J. Adrian, H. Aref, and D. J. Beebe, J. Microelectromech. Syst. 9, 190 (2000).

${ }^{2}$ C. C. Hong, J. W. Choi, and C. H. Ahn, Lab Chip 4, 109 (2004).

${ }^{3}$ N.-T. Nguyen and Z. Wu, J. Micromech. Microeng. 15, R1 (2005).

${ }^{4}$ V. Hessel, H. Löwe, and F. Schönfeld, Chem. Eng. Sci. 60, 2479 (2005).

${ }^{5}$ R. B. Bird, W. E. Stewart, and E. N. Lightfoot, Transpot Phenomenon, 2nd ed. (Wiley, New York, 2007).

${ }^{6}$ R. F. Ismagilov, A. D. Stroock, P. J. A. Kenis, G. Whitesides, and H. A. Stone, Appl. Phys. Lett. 76, 2376 (2000).

${ }^{7}$ A. E. Kamholz and P. Yager, Biophys. J. 80, 155 (2001).

${ }^{8}$ S. W. Lee, D. S. Kim, S. S. Lee, and T. H. Kwon, J. Micromech. Microeng. 16, 1067 (2006).

${ }^{9}$ A. D. Stroock, S. K. W. Dertinger, A. Ajdari, I. Mezic, H. A. Stone, and G. M. Whitesides, Science 295, 647 (2002). 